Voix et Images

voixetimages

\title{
Réalisme merveilleux et autoreprésentation dans l'Amélanchier de Jacques Ferron
}

\section{Mary Ellen Ross}

Volume 17, numéro 1 (49), automne 1991

Louky Bersianik

URI : https://id.erudit.org/iderudit/200946ar

DOI : https://doi.org/10.7202/200946ar

Aller au sommaire du numéro

Éditeur(s)

Université du Québec à Montréal

ISSN

0318-9201 (imprimé)

1705-933X (numérique)

Découvrir la revue

\section{Citer cet article}

Ross, M. E. (1991). Réalisme merveilleux et autoreprésentation dans

l'Amélanchier de Jacques Ferron. Voix et Images, 17(1), 116-129.

https://doi.org/10.7202/200946ar d'utilisation que vous pouvez consulter en ligne.

https://apropos.erudit.org/fr/usagers/politique-dutilisation/ 


\title{
Réalisme merveilleux et autoreprésentation dans l'Amélanchier de Jacques Ferron
}

\author{
par Mary Ellen Ross, Université de Victoria
}

L'Amélanchier de Jacques Ferron relève d'une double isotopie: ce roman peint, comme de nombreux récits de Ferron, des éléments surnaturels dans un cadre familier et reconnaissable. Les épisodes de l'Amélanchier se déroulent ainsi à Longueuil durant les années 1950 et $1960^{1}$. Cependant, à travers l'action du roman, les limites séparant l'humain, l'animal et le végétal s'estompent. Tinamer de Portanqueu se métamorphose en bécasse (p. 69-72) et les arbres du petit bois qui se trouve derrière sa maison présentent des caractéristiques humaines (p. 28-32). De même, le roman bouleverse systématiquement les catégories du temps et de l'espace: Tinamer peut ainsi rencontrer dans le petit bois de Longueuil un ecclésiastique mort à Québec en 1847 (p. 106-111).

Les spécialistes n'ont pas manqué de signaler la dimension "merveilleuse " de l'Amélanchier. Jean Marcel évoque son atmosphère de rêve et de fantaisie où tout est permis ${ }^{2}$. Jean-Pierre Boucher note lui aussi la présence du merveilleux dans l'Amélanchier:

Les arbres ne sont plus des choses inanimées mais des êtres vivants capables de parler, d'éprouver des émotions, doués d'une personnalité propre. Les animaux deviennent les frères des hommes; les actions des hommes eux-mêmes, mêmes les plus banales, sont investies par le merveilleux. 3

Cependant, il reste à situer l'Amélanchier dans l'ensemble assez vaste de textes théoriques traitant des genres dépourvus de validation

1 A titre d'exemple, les notes qui accompagnent une édition récente de l'Amélanchier repèrent dans le roman plusieurs références à des personnes habitant Longueuil durant les années 1950 et 1960 (Jacques Ferron, l'Amélanchier, édité par Pierre Cantin, Marie Ferron et Paul Lewis, Montreal, VLB éditeur, 1986, p. 165-173 [Courant]).

2 Jean Marcel, Jacques Ferron malgré lui, Montréal, Parti pris, 1978, p. 174.

3 Jean-Pierre Boucher, Jacques Ferron au pays des amélanchiers, Montréal, Presses de l'Université de Montréal, 1973, p. 36. 
empirique ${ }^{4}$ tels que le fantastique, le merveilleux, la science fiction et le réalisme merveilleux ${ }^{5}$. Dans le présent article nous examinerons donc le réalisme merveilleux dans l'Amélanchier. Comme nous le verrons, le caractère double du réalisme merveilleux ferronien ne provient pas uniquement du paradoxe apparent que constitue l'événement surnaturel peint dans un cadre "réaliste". Le nombre et la variété des techniques autoréférentielles qui se manifestent dans l'Amélanchier nous obligent à reconnaître une autre dualité dans ce roman, à savoir celle de la représentation et de l'autoreprésentation, refus elle aussi du réalisme mimétique. En fait, même si les spécialistes n'assignent guère la rubrique * réalisme merveilleux * aux récits de Ferron, la parenté que Victor Lévy-Beaulieu signale entre l'œuvre de Ferron et certains romans latino-américains ${ }^{6}$. suggère l'utilité, pour l'analyse de l'Amélanchier, d'un modèle qui tient compte et de la représentation du surnaturel et de l'autoreprésentation.

De loin la majorité des définitions qu'ont formulées les spécialistes du récit fantastique ou merveilleux se fondent sur la représentation d'épisodes surnaturels. Todorov insiste sur le caractère représentatif du discours littéraire fantastique, caractère qui autoriserait la distinction générale entre la fiction et la poésie ${ }^{7}$. Si Todorov reconnaît en passant que la fiction ne saurait relever entièrement de la représentation, comportant forcément des aspects *opaques", il considère: tout de même l'opacité du langage poétique comme une menace pour le fantastique:

Si, en lisant un texte, on refuse toute représentation et que l'on considère chaque phrase comme une pure combinaison sémantique, le fantastique ne pourra apparaître: il exige [...] une réaction aux événements tels qu'ils se produisent dans le monde évoqué. Pour cette raison, le fantastique ne peut subsister que dans la fiction; la poésie ne peut être fantastique (bien qu'il y ait des anthologies de "poésie fantastique»...). Bref, le fantastique implique la fiction. (p. 65)

4 La formule est de Darko Suvin, Pour une poétique de la science-fiction: études en théorie et en histoire d'un genre littéraire, Montréal, Presses de l'Université du Québec, 1977, p. 2. On peut désigner ces genres non mimétiques sous la rubrique générale du récit du surnaturel.

5 Jusqu'ici on n'a pas consacré d'analyse approfondie a l'usage que fait l'œuvre ferronienne du surnaturel. André Dallaire se sert du modàle todorovien du fantastique pour étudier le conte * Le chien gris * ( Le "Fantastique" Ferron*, Arches, $\mathrm{n}^{\circ} 1$, printemps 1973, p. 34-42) $\AA$ part cet article, la critique québécoise n'a pas confronté l'œuvre ferronienne et les théries du fantastique, du merveilleux et du réalisme merveilleux.

6 Victor Lévy-Beaulieu, «Roman des Amériques: la grande leçon de José Donoso et G. G. Marquez aux romanciers québécois *, le Devoir, vol. LXIV, $\mathrm{n}^{\circ}$ 207, 8 septembre 1973, p. 15.

7 Tzvetan Todorov, Introduction à la littératiure fantastique, Paris, Seuil, 1970, p. 64. 
D'autres spécialistes soulignent l'importance, pour le récit du surnaturel, de la représentation non ambiguë de ce qui brise les lois de la réalité empirique. En fait, le terme même de "surnaturel" suppose l'aptitude du texte à évoquer des épisodes qui sont incompatibles avec la causalité de la réalité extratextuelle. W.R. Irwin représente le point de vue selon lequel le fantastique utiliserait les techniques du réalisme: [...] fantasy is bound to realistic traditions and methods ${ }^{8}$. Pour des critiques comme Irwin, le récit du surnaturel met en œuvre les procédés de la représentation réaliste, pour évoquer ce qui est manifestement irréel, voire impossible.

Pourtant, les travaux de Irlemar Chiampi proposent une hypothèse tout autre. La narration du réalisme merveilleux se caractérise par une causalité à la fois diffuse et non problématisée ${ }^{9}$. Mais ce réalisme merveilleux, où coexistent la représentation de la réalité empirique et celle d'épisodes surnaturels, constitue pour Chiampi une mise en question moins de la nature de la réalité que de l'aptitude de la fiction à représenter cette réalité extra-textuelle (p. 101). À son avis, les ouvrages les plus typiques du réalisme merveilleux font preuve d'une mise en relief du narrateur et de sa situation, à la différence du roman réaliste, qui occulterait l'acte et la situation d'énonciation (p. 85-88). L'autoreprésentation serait donc centrale au réalisme merveilleux.

L'Amélanchier est plus conforme au modèle de Chiampi qu'à ceux formulé par Todorov et Irwin ${ }^{10}$. Lintrication dans ce roman de la représentation du surnaturel et de l'autoreprésentation, double refus du réalisme mimétique, est telle que l'analyse d'un seul de ces aspects du texte ne saurait rendre compte du discours réalistemerveilleux ${ }^{11}$ ferronien.

8 W.R. Irwin, The Game of the Impossible: A Rhetoric of Fantasy, Chicago, University of Mlinois Press, 1976, p. 188. Traduction: [...] le fantastique est lié aux traditions et aux méthodes réalistes.

9 Irlemar Chiampi, El realismo maravilloso: forma e ideologia en la novela hispanoamericana, Caracas, Monte Avila Editores, 1983, p. 71-72. Chiampi signale que, si les rubriques "réalisme magique" et "réalisme merveilleux * s'emploient pour désigner un même corpus de romans, cette dernière est tout de même préférable, dans la mesure où le terme " merveilleux * relève de la terminologie littéraire (p. 49).

10 Ce n'est certes pas la première fois que l'on rapproche le roman québécois et le roman latino-américain. A titre d'exemple, Amaryll Chanady note certains traits autoreprésentatifs que partagent l'œuvre d'Hubert Aquin et celle, souvent qualifié de réaliste-merveilleuse ou magico-réaliste, de Julio Cortázar (Amaryll Chanady, * Entre la quête et la métalittérature - Aquin et Cortázar comme représentants du postmoderne excentrique *, Voix et images, $n^{\circ} 36$, automne 1986, p. 42-53).

11 Nous suivons l'exemple de Maximilien Laroche et de Cecilia Ponte en employant l'adjectif « réaliste-merveilleux * pour qualifier le réalisme mervieilleux (Maximilien Laroche; Contributions à l'étude du réalisme merveilleux, Québec, Grelca, 1987 [Essais n 2]; Cecilia Ponte, Le Réalisme merveilleux dans * Les Arbres musiciens » de Jacques-Stéphen Alexis, Québecc, Grelca, 1987 [Essais, n 1]) 
Nous examinerons l'autoreprésentation dans l'Amélanchier à partir des trois plans généraux proposés par Janet Paterson, ceux de l'énonciation narrateur, de l'énoncé narration et de l'énonciation narrataire. Comme le note Paterson, ces catégories, différenciées dans le modèle et sur le plan de l'analyse sont fréquemment imbriquées dans la pratique du texte littéraire 12 . Il ne nous sera donc pas toujours possible, ni même souhaitable, de suivre fidèlement ce schéma, qui servira plutôt de guide dans cette lecture de l'Amélanchier.

On remarque tout de suite, en abordant l'Amélanchier, que le roman fait preuve d'éléments autoréflexifs sur le plan de l'énonciation. La narratrice déclare dès l'incipit le but de sa démarche: Mon enfance je décrirai pour le plaisir de me la rappeler, tel un conte devenu réalité, encore incertaine entre les deux (p. 27). On a bel et bien affaire à un personnage écrivant dans l'Amélanchier, un personnage qui rédige en fait le roman. Comme le précise Paterson, la mise en scène d'un personnage écrivain relève d'une si longue tradition littéraire qu'il est aisé d'y voir un reflet de l'activité de la création artistique (p. 179). Il s'agirait selon l'incipit d'une Tinamer adulte qui assume la narration du récit et qui ne prétend nullement offrir de son enfance un récit fidèle et "vrai *: cette narration aura le caractère d'un conte devenu réalité. La narratrice avertit le lecteur que la narration se situe à michemin entre la " réalité * référentielle et la fiction pure ${ }^{13}$.

La narratrice a recours à d'autres procédés pour signaler que son récit n'est pas "réel». À l'avant-dernier chapitre du roman, on retrouve la confusion flagrante de l'histoire personnelle supposément vécue et de l'écriture:

Dans ma solitude, j'éprouve du regret pour un conte inachevé, pour la quiétude et le bonheur qu'il représentait. J'ai même l'impression d'en avoir été chassée. C'était un beau conte hétéroclite où à ma mémoire s'ajoutaient la vôtre, celle aussi de Bélial, de Thibeau, de Jaunée et de Bouboule... [...] Et je me

12 Janet Paterson, "L'autoreprésentation: formes et discours ", Texte, $\mathrm{n}^{\circ} 1,1982$, p. 178. De même, Lucien Dällenbach démontre, dans sa tentative d'esquisser une typologie de la mise en abyme, le recouvrement qui caractérise forcément toute distinction entre éléments qui relèvent de l'énoncé et ceux qui appartiennent à l'énonciation ou au code. (Lucien Dăllenbach, le Récit spéculaire: escai sur la mise en abyme, Paris, Seuil, 1977, p. 139.)

13 Catherine Kerbrat-Orecchioni présente une théorie de la réception du texte litterraire qui correspond d'assez près à l'expression qu'emploie la narratrice de l'Amélanchier. Kerbrat-Orecchioni soutient que le texte littéraire n'est autre qu'un gigantesque trope fictionnel, lequel instaure [...] un décalage entre le paraitre discursif (le reférent existe pour de vrai) et l'etre discursif (le reférent n'existe que "pour de faux»). ("Le texte littéraire: non-référence, auto-référence, ou référence fictionnelle? », Texte, $n^{\circ} 1,1982, p .39$.) Vue à la lumière de l'article de Kerbrat-Orecchioni, l'assertion de la narratrice fonctionne comme une sorte d'embrayeur de fictionalite (p. 41). 
dis en cette captivité de mes vingt ans, je me dis Tinamer, ma pauvre Tinamer, tu as lancé un peu vite, il me semble, les bouledozeurs en arrière de la maison, dans le petit bois enchanté et bavard. (p. 145)

La narratrice commence par évoquer le *conte " qu'elle vivait avec ses parents, ses chats et son chien, conte dont il lui semble avoir été chassée. Tinamer avoue ne pas contrôler elle-même le déroulement du récit, dans la mesure où elle se sent expulsée de l'histoire où elle prétendait figurer comme personnage.

Un deuxième passage tiré de l'avant-dernier chapitre de l'Amélanchier est essentiel à l'analyse de l'autoreprésentation sur le plan de l'énonciation:

Me voici seule dans ma chambre, la plume à la main, assise à une table encombrée des pages que jai déjà écrites et des livres dont je me suis inspirée, Ollivier, épopée chevaleresque de Cazotte, Les Bois-Francs, de l'abbé Charles-Edouard Mailhot, dont j'ai tiré, sans guère y changer, le saint personnage de. Messire Hubert Robson, et Le Sens de la direction et de l'orientation chez l'homme, du docteur ès-lettres Pierre Jaccard. Sur ce dernier ouvrage, je garde, encore incongrue, la boussole de Monsieur Northrop. Une boussole qui fait wasp. Pour un ancien lapin domestiqué, dressé, discipliné, devenu wasp lui-même, c'était un fétiche indispensable. (p. 147)

Aux commentaires métafictionnels signalés dans le passage cité cidessus s'ajoute ici un autre trait autoréflexif: la narratrice fait connaître les sources du récit, les autres textes dans lesquels elle aurait puisé certains éléments du roman. L'intertextualité constitue selon Paterson un procédé capital du redoublement textuel qui se situe au niveau du code ${ }^{14}$. Ici, le redoublement textuel est flagrant, dans la mesure où la narratrice signale explicitement l'intertexte, sans laisser au lecteur la tâche de lidentifier. Les conséquences qu'entraîne pour le surnaturel cette démarche de la part de la narratrice sont capitales: dans la mesure où certains épisodes qui semblaient relever du surnaturel sont attribués à d'autres ouvrages, le texte signale le caractère emprunté, voire * faux * de ce surnaturel. Du moment où la narratrice prétend s'être inspirée de l'ouvrage de l'abbé Mailhot pour créer le personnage d'Hubert Robson, la rencontre merveilleuse de cet ecclésiastique (mort depuis plus d'un siècle) et de Tinamer risque de n'être qu'une pure convention textuelle. Il en va de même de l'intertexte, cette fois-ci moins explicite, d'Alice au pays des merveilles. Comme Boucher le reconnaît ${ }^{15}$,

14 Janet Paterson, loc. cit., p. 184.

15 Jean-Pierre Boucher, op. cit., p. 87. 
certains épisodes et personnages de l'Amélanchier ressemblent de près à ceux que présente l'ouvrage de Lewis Carroll: comme Alice, Tinamer semble s'endormir sur lherbe, suivre un lapin anthropomorphique, rapetisser (p. 57-66). De plus, Monsieur Northrop ${ }^{16}$ consulte à tout moment sa boussole, geste qui rappelle celui du lapin de Lewis, qui sortait souvent sa montre. Ce faisant, précise Léon, Monsieur Northrop se souvient de tout, de son existence antérieure au pays des merveilles (p. 34). Cet intertexte met en évidence le caractère non réaliste de certains épisodes de l'Amélanchier, tout en mettant en question la "réalité" de ces épisodes: après tout, les "merveilles" racontées dans Alice au pays des merveilles ne sont en fin de compte que des épisodes du rêve de la jeune protagoniste.

Ainsi; le passage confond volontiers illusion et réalité, comme le font les deux autres extraits du roman que nous avons cités. Messire Hubert Robson est un personnage tiré d'un autre ouvrage, tandis que Tinamer adulte garde toujours la boussole de Monsieur Northrop, ancien lapin domestiqué, dressé, discipliné. Monsieur Northrop aurait "réellement " existé, c'est-à-dire qu'il aurait été un personnage qui fréquentait le bois pendant l'enfance de Tinamer. En même temps; la narràtrice met en question l'existence de ce personnage en commentant le caractère approprié de la boussole comme accessoire: en soulignant que la boussole est un fétiche indispensable à ce personnage, la narratrice semble indiquer qu'il ne s'agit dans le cas de la boussole que d'une invention. Le cas de la boussole est représentatif de l'impossibilité de dissiper l'équivoque créée par le passage. L'intertexte explicite met en question, par le biais de l'autoreprésentation, la référentialité de certains éléments du texte, tandis que le texte par ailleurs présente comme « réels * les personnages et les épisodes que la narratrice aurait tirés des ouvrages signalés.

Lorsqu'on passe à l'étude de l'autoreprésentation sur le plan de l'énoncé, on remarque le nombre considérable de mises en abyme que contient le roman ${ }^{17}$. La première mise en abyme peint Tinamer, perdue dans le bois, qui semble dormir et qui se métamorphose en oiseau ${ }^{18}$. Les chapitres qui suivent le "rêve " de Tinamer présentent

16 On constate également ici, dans la mesure ou le personnage de Monsieur Northrop s'inspire en partie de Northrop Frye, célèbre théoricien littéraire, un lien autoréférentiel entre la fiction de l'A mélanchier et' la théorie littéraire. (Voir Jacques Ferron, l'Amélanchier, op. cit., p. 165, note 2)

17 Boucher consacre une partie de son analyse de l'Amélanchier à la mise en abyme, qui aurait, comme la fragmentation du recit, 'la fonction de rompre le caractère linéaire du récit, assimilé au labyrinthe, et de lui donner une forme à l'imagne [sic] du monde merveilleux de l'enfance (p. 93).

18 Encore une fois, la narratrice met en question la véracité des épisodes racontés. Après avoir décrit sa propre transformation merveilleuse, Tinamer se demande si cet incident n'a pas été un réve: Me serais-je endormie au début de mon rêve [...]? (p. 72) 
de nouvelles mises en abyme. Le sixième chapitre contient un récit où Léon raconte, d'abord à la troisième personne et ensuite à la première personne, son enfance. Cette dernière mise en abyme contient de nombreuses réduplications, au moyen desquelles le texte se présente explicitement comme tel ${ }^{19}$.

La narration par Léon insiste sur les caractéristiques qu'il partage avec Tinamer:

Quand j'avais ton âge, Tinamer, il y avait dans le comté de Maskinongé un petit garçon qui te ressemblait beaucoup. [...]

Il avait les cheveux de ta couleur, à peu près de la même longueur, mais alors que tu penses les avoir aussi courts qu'un garçon, il pensait les avoir aussi longs qu'une fille. (p. 73)

Ce dédoublement de Léon et de Tinamer continue tout au long du passage: la description que fait Léon de la première nuit qu'il a passée, enfant, sans oublier qui il était, reflète la situation de Tinamer, qui éprouve elle aussi le besoin de ne pas perdre son identité lorsqu'elle s'endort la nuit ${ }^{20}$. En fait, Tinamer se demande si son propre rapetissement, ainsi que sa métamorphose en bécasse, ne sont pas la contrepartie d'une nuit de l'enfance de Léon, racontée par ce dernier: En écoutant Léon parler ainsi, je me demandais si la nuit que javais rêvée, autrement plus spectaculalre que son incendie d'église, n'était pas l'équivalent de la sienne (p. 79). La réduplication qui rapproche Tinamer et Léon met en question le statut des épisodes apparemment surnaturels vécus par Tinamer, en insistant sur leur caractère multiple, universel et même habituel.

Le septième chapitre de l'Amélanchier offre un extrait de la "bible des de Portanqueu", mise en abyme qui relève évidemment de l'intertextualité. Le narrateur de cette "bible" souligne même en quoi elle ressemble à la bible de tout le monde, et en quoi elle s'en écarte: Le péché d'origine ne retrouve pas [sic] dans la complexion québécoise. Les de Portanqueu n'ont pas recours à un père et à une mère incestueux: ils tirent leur origine de trois frères. (p. 82)

La mise en abyme de la bible des de Portanqueu contient d'autres éléments autoreprésentatifs. Paterson reconnaît, sans

19 Sous la rubrique générale de la diégèse Paterson signale, après la catégorie des mises en abyme, celle des réduplications. Il s'agit des repétitions, des doublures, des enchassements ou des reflets qui, d proprement parler, ne sont pas des mises en abyme (op. cit., p. 181).

20 Le parallèle est explicite: Tinamer commente le récit en abyme de Léon en disant que ce dernier n'a raison que sur un point, le manque de mémoire des enfants. On oublie de jour en jour ce qu'on est (p. 77). D'ailleurs, une autre mise en abyme, celle du dossier de l'enfant aliéné Jean-Louis Maurice, reprendra la question de la perte d'identité de l'enfant qui s'endort (p. 139-140). 
poser en équivalence le narrateur et l'auteur ou bien le narrataire et le lecteur, [...] [une] homologie qui gouverne à la fois les principales articulations dans le texte et la visée du discours interprétatif. ${ }^{21}$

Le récit des trois frères dans le septième chapitre de l'Amélanchier, qui est la suite de la bible des de Portanqueu, nous oblige à reconnaître cette homologie partielle du narrateur et de l'auteur.

Il s'agit dans le récit des trois frères de la lignée des de Portanqueu, famille dont les ancêtres auraient été des Ferron: C'est là [dans le comté de Maskinongé] que commence l'histoire des de Portanqueu. Longtemps ils n'en menèrent pas large. Ils s'appelaient Ferron. (p. 84) Le narrateur de la mise en abyme continue à tracer l'histoire des Ferron, jusqu'au moment d'aborder le récit des trois frères :

La légende des trois frères descendant du déluge pour fonder Machiche, les Ferron se la sont appropriée, quitte à se mettre dans l'embarras de ne pas savoir duquel des deux ils descendaient, n'étant sûrs que du troisième, prénommé Jean, qui n'avait pas fait souche de Ferron en pays machinois, ayant continué l'aventure [...]. (p. 85)

Il est difficile de ne pas reconnaitre en cet endroit du texte l'empreinte de l'auteưr réel, rappel des liens qu'entretient celui-ci avec l'histoire et les personnages qu'il crée.

L'Amélanchier présente d'autres mises en abyme, notamment celle où Messire Hubert Robson raconte à Tinamer sa propre mort, épisode qui relève franchement du surnaturel (p. 106:111). Cependant, dans ce dernier cas, comme dans ceux que l'on a déjà étudiés, la narration du surnaturel n'est point celle, linéaire et simple, décrite par Irwin. L'important élément autoreprésentatif, présent dans la mise en abyme, confere à cette narration un caractère complexe, soulignant à tout moment son statut de création littéraire, de " texte *.

Lorsqu'on passe de la mise en abyme aux réduplications, on note encore la richesse d'éléments autoreprésentatifs dans l'Amélanchier. Il faudrait surtout relever les dédoublements de personnages, procédé qui permet au récit de présenter, dans l'Amélanchier, un personnage du même nom qu'un personnage qui figure ailleurs dans l'œuvre de Ferron.

Le cas de Tinamer, narratrice de l'Amélanchier, est assez typique de ce phénomène autoréflexif, très répandu chez Ferron. Nous trouvons ailleurs dans son œuvre des personnages baptisés Tinamer, dont la fille du premier Jean Goupil dans la Chaise du

21 Janet Paterson, op. cit., p. 178. 
maréchal ferrant et la femme du deuxième Jean Goupil dans ce même roman ${ }^{22}$. Le jeu des réduplications est évidemment complexe: le premier Jean Goupil a également des filles qui s'appellent Marie et Chaouac. Ce dernier nom est celui d'un autre personnage féminin que l'on trouve chez Ferron, dans "La vache morte du canyon" 23 . Ainsi, les noms qui figurent dans ces textes renvoient les uns aux autres, formant un réseau de reflets qui ne se limite pas à l'Amélanchier. En dépit de la complexité de ces rapports, leur effet est simple: il s'agit encore une fois de mettre l'accent sur le texte comme création littéraire ${ }^{24}$.

Reste à examiner une dernière catégorie signalée par Paterson, qui permet au récit de rappeler son propre statut fictif. L'Amélanchier est riche en éléments autoréflexifs au niveau du champ lexical. Monsieur Northrop est un "figurant» du bois enchanté qui, se trouve derrière la maison des de Portanqueu (p. 34). De même, la narratrice souligne le statut de personnage littéraire d'Etna:

Lors de mes premières années, fort obscures et confinées, dont j'ai oublié la lactation et les couches, la lumière plate qui bouchait l'espace et les trous qu'y pratiquait ma mère pour me faire apparaître la madone qui calmait mes pleurs et me nourrissait [...]. (p. 36)

Comme la madone des tableaux religieux, la mère de Tinamer "apparaît» en tant que représentation ${ }^{25}$. D'ailleurs, la narratrice généralise plus loin:

Les adultes, vilains comédiens jouant toujours le même rôle, ne comprennent pas que l'enfance est avant tout une aventure intellectuelle où seules importent la conquête et la sauvegarde de l'identité, que celle-ci reste longtemps précaire et que, tout bien considéré, cette aventure est la plus dramatique de l'exis-

22 Jacques Ferron, la Chaise du maréchal ferrant, Montréal, Éditions du Jour, 1972.

23 Jacques Ferron, "La vache morte du canyon", Contes, Montréal, HMH, 1968, p. 74-98. La présence de l'auteur réel risque de s'imposer par les noms de personnages, pour quiconque sait que Ferron lui-même a eu trois filles, nommées Anne (surnommee Chaouac), Marie et Martine (dont l'anagramme est Tinamer).

24 D'autres noms de personnages de l'Amélanchier trouvent leur contrepartie ailleurs dans l'cuvre de Ferron. Jean-Louis Maurice, baptisé Coco, rappelle le perroquet Coco du conte "Le perroquet* (Contes, op. cit., p. 51-55). Il est question dans l'Amélanchier de Rédempteur Fauché (p. 46, 96) et de Papa Boss (p. 46, 48, 105). Rédempteur Fauché est un personnage du Ciel de Québec, tandis que Papa Boss a donné son nom à un autre récit ferronien (Jacques Ferron, le Ciel de Québec, Montréal, VLB éditeur, 1979; Papa Boss dans les Confitures de coings et autres textes, Montréal, Parti pris, 1977).

25 Léon, de même, est décrit comme un personnage imaginaire de la commedia dell'arte: le père de Tinamer est comme un grand Polichinelle sorti de l'ombre (p. 52). 
tence. Les cabotins l'ont oublié et font des livres stupides pour avantager le petit. rôle de leur minable personnage. (p. 63)

Tout au long de ce passage autoreprésentatif, la narratrice confond volontiers la vie « réelle» des personnages du récit et la fiction. Les adultes sont des " comédiens ", l'enfance est une aventure [...] dramatique, et des auteurs valorisent le "rôle " de leur propre "personnage" dans un livre. Le champ lexical attire ainsi l'attention sur le caractère littéraire du récit, comme le font les mises en abyme et les réduplications.

En plus de signaler son propre caractère général de fiction, l'Amélanchier se présente explicitement comme récit réalistemerveilleux. Il s'agit d'un trait autoreprésentatif présent au niveau du code, et plus précisément, d'un paradigme particulier du champ lexical du récit littéraire réaliste-merveilleux.

Cecilia Ponte, qui étudie le réalisme merveilleux du romancier haïtien Jacques-Stéphen Alexis, consacre une partie de son étude à l'emploi alexisien de vocables autoreprésentatifs du réalisme merveilleux. La dernière partie de ce tour d'horizon de l'autoreprésentation dans le réalisme merveilleux de l'Amélanchier traitera de la tendance du récit à signaler non pas son propre caractère général de fiction, mais plus précisément son caractère de fiction réaliste-merveilleuse.

Ponte signale l'importance de ce procédé d'«embrayeurs génériques " pour le système descriptif dans le réalisme merveilleux:

certains termes (souvent des adjectifs) indiquent au lecteur son entrée dans le merveilleux. En tant qu'embrayeurs génériques, ils lui signalent qu'il assiste à une description réalistemerveilleuse et en commandent un certain décodage. [...] Ainsi, par leur distribution et leur abondance, les signaux autoréférentiels se rendent garants du réalisme merveilleux en texte. ${ }^{26}$

L'Amélanchier offre un nombre élevé de termes tels que "enchanté» et "merveilleux", qui mettent en relief le caractère réaliste-merveilleux du récit. De fait, nous avons compté quaranteneuf de ces vocables dans ce texte, susceptibles de regroupement en quelques constellations.

L'Amélanchier propose de nombreux adjectifs qui mettent l'accent sur l'incongruité des épisodes, du décor et des personnages du roman. À titre d'exemple, le vieux gentleman britannique est décrit comme le curieux Monsieur Northrop (p. 79; nous soulignons, ainsi que dans les citations à venir). Ce même personnage fait remarquer

Cecilia Ponte, op cit., p. 63. 
deux fois à Tinamer que celle-ci vient d'émettre un curieux raisonnement (p. 96). Même la banlieue de Montréal se présente comme une incroyable municipalité (p. 36).

L'adjectif «enchanté * paraît huit fois au cours du récit ${ }^{27}$. Cet adjectif qualifie inévitablement le bois qui se trouve derrière la maison de Tinamer. On retrouve ce qualificatif dans cinq passages tirés des deux premiers chapitres du récit, dans la bouche de la narratrice aussi bien que dans celle de Léon. Lorsque l'adjectif * enchanté * paraît de nouveau, dans le dixième chapitre du récit, la narratrice l'emploie de façon ironique pour marquer la fin de sa première enfance. Ce chapitre présente le désenchantement de Tinamer ${ }^{28}$, lorsque la fillette apprend que le monde n'est pas tel que son père le lui avait décrit:

Si nous avions pu descendre sur la grève de cette merveille de la nature, nous aurions constaté que le majestueux SaintLaurent était gras d'eau de vaisselle, qu'il puait, égout à ciel ouvert, égout de tous les égouts du Haut et du Bas-Canada, et qu'il charriait des étrons, des étrons à n'en, plus finir, avec, ça è là, la charogne d'un nègre américain assassiné à Chicago encore chanceux que le Mékong ne soit pas un affluent de notre fleuve national et biculturel.

Au-delà, nous pouvions apercevoir les quais de Montréal, quelques cargos, de grands silos et le quartier Hochelaga derrière comme une sorte de désert rouge. C'était tout ce que nous trouvions en face de nous, au bout du bois enchanté et bavard. (p. 121)

Le substantif *merveille» s'emploie également de façon ironique dans ce passage.

Comme c'était le cas de l'adjectif "enchanté", le qualificatif "merveilleux" et le substantif "merveille* ne s'emploient pas de façon ironique dans les chapitres qui précèdent le désenchantement de la fillette. Dans le premier chapitre, Léon se réfère à l'existence antérieure de Monsieur Northrop * au pays des merveilles ", évoquant ainsi l'intertexte qu'est l'ouvrage de Lewis Carroll (p. 34). Et lorsque Léon prend en charge la narration dans le sixième chapitre, il se réfêre à un acte assez banal comme la merveille du genre humain (p. 73).

Mais au moment où Tinamer apprend ce qui se trouve au bout du bois * enchanté», c'est-à-dire la ville de Montréal et non pas, comme

27 L'Amélanchier, p. 28, 30, 31, 34, 38, 121, 145 et 153.

28 Le terme * désenchantement * est juste dans le contexte, puisque Tinamer répudie provisoirement le bois « enchanté *. 
Léon l'avait prétendu, le comté de Maskinongé, les vocables qui désignent le réalisme merveilleux s'emploient de façon ironique, ou bien se réferent au passé de Tinamer, avant la découverte décisive. Ainsi, le bois avait été le prodigieux domaine de Tinamer (p. 120), Léon avait cessé de faire le magicien (p. 122), la merveille de la nature est un fleuve pollué, les merveilles de l'île Sainte-Hélène sont de grands buildings de verre et d'acier (p. 121). Comme on s'y attendrait, les embrayeurs génériques du réalisme merveilleux ne figurent plus de façon simple et directe lors du rejet par Tinamer du merveilleux.

La disette de termes autoreprésentatifs du réalisme merveilleux dans le onzième chapitre de l'Amélanchier est révélatrice à cet égard. Il est question dans ce chapitre de la distance qui sépare Tinamer de son père après la première enfance de la fillette et du dossier médical d'un enfant interné, Jean-Louis Maurice, dit Coco. La rareté des termes autoréférentiels du réalisme merveilleux correspond donc et à l'absence d'épisodes surnaturels dan's ce chapitre, où l'adolescente Tinamer s'éloigne de son père, et au caractère «scientifique » du dossier médical de Coco.

Cependant, la formule des deux premiers chapitres figure de nouveau dans le dernier chapitre de l'Amélanchier; la narratrice prétend voir de nouveau le bois enchanté et bavard (p. 153). Ainsi, le texte se représente en fin de compte explicitement comme une narration réaliste-merveilleuse.

L'Amélanchier se caractérise donc par l'abondance de termes emblématiques tels que "merveilleux * et * enchanté», qui rappellent constamment qu'il s'agit d'un récit réaliste-merveilleux. Comme le soutient Paterson, l'importance de l'autoréflexivité se reconnaît à la présence de traits autoreprésentatifs à plusieurs niveaux du texte, ainsi qu'à une certaine redondance, à un processus d'accumulation qui régit la pratique signifiante (p. 183). Il est aisé de constater cette redondance en ce qui concerne les termes dans l'Amélanchier qui signalent le caractère réaliste-merveilleux du récit, d'autant plus que ces vocables renvoient souvent à des épisodes assez courants qui ne relèvent pas du surnaturel.

Cette lecture des principaux procédés de l'autoreprésentation dans l'Amélanchier, loin d'être exhaustive, démontre néanmoins l'étendue du phénomène. La multiplicité et l'interdépendance des techniques autoréférentielles dans ce récit sont frappantes. L'Amélanchier propose de nombreux commentaires faits par la narratrice sur le récit qu'elle présente et sur les récits internes mis en abyme, qui reprennent les éléments du récit noyau. Les réduplications érigent dans l'Amélanchier un système de reflets, qui évoquent, comme le font l'intertextualité et le champ lexical du récit, son caractère fictionnel et réaliste-merveilleux. 
Pour Irwin, qui mesure la réussite du récit du surnaturel à partir de la présentation convaincante et réaliste d'épisodes irréels, l'Amélanchier de Ferron serait une tentative vouée à l'échec. Mais un tel jugement supposerait que l'emploi de traits autoréférentiels provienne d'une maladresse ou d'une erreur de la part de l'auteur. Compte tenu de l'emploi régulier de ces techniques dans l'Amélanchier, il est difficile de souscrire à cette opinion. La représentation d'éléments surnaturels s'accompagne inévitablement dans ce récit de la mise en évidence systématique de la littérarité du texte.

Faut-il donner raison à Irwin lorsque ce critique considère que le récit du surnaturel doit n'employer que des techniques du réalisme? Certes, comme le signale Paterson, il existe une opinion critique selon laquelle l'autoréflexivité constitue un danger pour l'illusion référentielle ${ }^{29}$. Ricardou est au nombre de ceux qui conçoivent l'autoreprésentation comme une contestation de la fonction représentative du discours littéraire: Tous les efforts que fait la fiction pour représenter la narration qui l'engendre s'accomplissent au détriment de toute éventuelle représentation $d u$ monde ${ }^{30}$. Les tenants de cette thèse estiment que l'autoreprésentation, menace pour la représentation, l'est doublement dans le cas d'un récit qui présente des épisodes surnaturels: selon Irwin, plus l'adhésion du lecteur est difficile à obtenir, plus le texte doit s'efforcer d'obéir aux conventions du réalisme. Pourtant, cet avis ne fait pas l'unanimité parmi les spécialistes du récit du surnaturel ni parmi ceux de l'autoreprésentation.

Paterson suggère la possibilité d'

une corrélation entre la représentation et l'autoreprésentation. Dans certains romans de Nabokov, Gide, Proust, Butor; Hébert, Aquin et Barth (c'est-à-dire dans une écriture moderne qui ne répudie pas entièrement la représentation), on observe justement une intégration maximale de ces deux systèmes. Par ses formes et par ses thèmes; l'autoreprésentation participe à la production du sens au niveau de la fiction alors que la représentation dirige notre lecture vers la découverte du fait littéraire. ${ }^{31}$

Certains critiques qui étudient la littérature du surnaturel affirment, de même, que la représentation et l'autoreprésentation ont partie liée. Linda Hutcheon estime en fait que la littérature fantastique offre un modèle particullèrement approprié à la métafiction:

The invented autonomous cosmos of fantasy becomes a model in metafiction for the temporal and spatial structuration of

29 Janet Paterson, op. cit., p. 191-192.

30 Cité par Janet Paterson, ibid., p. 191.

31 Janet Paterson, ibid., p. 192-193. 
both the universe of the work and its very narration - and this is so from the perspectives of both writer and reader. ${ }^{32}$

La construction d'une réalité secondaire, non empirique, serait emblématique de la création littéraire et de l'acte d'écrire en général.

Il en est ainsi dans l'Amélanchier: la dissolution des frontières du naturel et du surnaturel se joint, dans le roman, à la mise en relief de l'écriture. Ainsi, le réalisme merveilleux dans l'Amélanchier n'est ni le miroir de la réalité empirique ou d'une réalité secondaire et magique, ni, non plus, un miroir de lui-même, mais la combinaison de tous ces éléments en apparence incompatibles, qui font la richesse et la complexité du récit réaliste-merveilleux chez Ferron.

32 Linda Hutcheon, "Narcissistic Narrative: The Metafictional Paradox", Canadian Review of Comparative Literature, $n^{\circ} 5,1980$, p. 78. Traduction: L'univers fictif et autonome du fantastique devient un modele de métafiction pour la structuration temporelle et spatiale d la fois de l'univers de l'auvre et de sa narration - et il en est ainsi pour les perspectives.à la fois de l'écrivain et du lecteur. 